\title{
Paradoxical Herniation in the Postcraniectomy Syndrome: Report and Literature Update
}

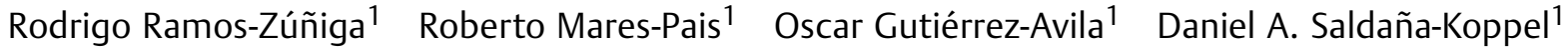 \\ 1 Department of Neurosciences, Universidad de Guadalajara, \\ Translational Neurosciences Institute, Guadalajara, Jalisco, Mexico \\ Address for correspondence Rodrigo Ramos-Zúñiga, Department of \\ Neurosciences, University of Guadalajara, Victoria 1531-4, \\ Guadalajara, Jalisco 44630, Mexico (e-mail: rodrigorz13@gmail.com).
}

J Neurol Surg Rep 2016;77:e35-e38.

\begin{abstract}
Keywords

- cerebral edema

- cranioplasty

- decompressive craniectomy

- paradoxical herniation

- sinking skin flap syndrome

- syndrome of the trephined

Introduction The decompressive craniectomy is a surgical strategy widely used with specific criteria to control the refractory intracranial pressure (ICP). However, it is important to warn about the presence of a postcraniectomy syndrome and analyze the risk-benefit on a long term.

Case Report A 72-year-old male patient diagnosed with a subarachnoid hemorrhage secondary to the rupture of an anterior circulation aneurysm that develops vasospasm, secondary ischemia, and edema with signs of herniation that required a decompressive craniectomy on a first step. Afterwards, the aneurysm was approached and he consequently developed hydrocephaly. A ventriculoperitoneal shunt is installed, contralateral to the craniectomy, and progressive sinking of the skin flap, there is neurological deterioration and paradoxical herniation. Its association with the clinical deterioration by bronchoaspiration did not allow the cranioplasty to resolve the ICP decompensation.

Conclusions The paradoxical herniation as part of the postcraniectomy syndrome is an increasingly common condition identified in adult patients with cortical atrophy, and who have also been treated with ventricular shunt systems. Timely cranioplasty represents the ideal therapeutic plan once the compromise from the mass effect has resolved to avoid complications derived from the decompressive craniectomy per se.
\end{abstract}

\section{Introduction}

Decompressive craniectomy is a valid neurosurgical strategy now a day as an alternative to control an elevated intracranial pressure (ICP) and controlling the risk of uncal and/or subfalcine herniation, in refractory cases to the postural, ventilator, and pharmacological measures to control it. The neurocritical care and the ICP monitorization are key determinants to identify and postulate the inclusion criteria to consider a patient as candidate to this procedure, as it is always considered a rescue surgical technique. Head trauma and ischemic or hemorrhagic cerebrovascular disease with progressive deterioration due to mass effect are some of the cases that may require a decompressive craniectomy with its different variants. However, this procedure per se can have complications described in the postcraniectomy syndrome and may occur in short, medium, or even long term. ${ }^{1,2}$ The paradoxical herniation is a condition in which there is a deviation of the midline with mass effect, even though the skull is not covered, which clinically relates to the sinking of the skin flap and the intracranial pressure changes that are more susceptible to the posture and the effect of atmospheric pressure. This herniation pattern is much more evident and susceptible to deterioration in cases where a ventricular shunt has also been installed, which contributes to a ventricular collapse and modification and alteration of the Monro-Kellie law, with the consequent maladjustment and neurological deterioration due to the paradoxical herniation. received

September 5, 2015

accepted

November 5, 2015

published online

January 4, 2016
DOI http://dx.doi.org/

10.1055/s-0035-1570349. ISSN 2193-6358. (c) 2016 Georg Thieme Verlag KG
Stuttgart · New York

License terms

(1) $\Theta \circledast$ 
This condition requires prompt treatment by cranioplasty and should be considered as part of the postcraniectomy syndrome, as it may be responsible for the neurological impairment and/or sudden death. ${ }^{3-6}$

\section{Case Report}

A 72-year-old male patient that presents headache, diminished state of consciousness, and left hemiparesis. A subarachnoid hemorrhage is identified secondary to a ruptured supraclinoid internal carotid aneurysm with associated ischemia in the right medial cerebral artery territory and cerebral edema. Decompressive craniectomy was performed, and in a second intention the clipping of the aneurysm. After 2 weeks, neurological deterioration is observed due to secondary hydrocephaly that required a ventriculoperitoneal shunt. His neurological condition persisted as grade IV in the Hunt and Hess scale and a gastrostomy was installed for feeding 10 days after installing the ventriculoperitoneal shunt, all of this done in a public sector hospital. Afterwards, gradual sinking of the skin flap was identified in the cranial defect and new neurological deterioration with a comatose patient and rigidity of decerebration, critical condition that did not allow the cranioplasty to be performed, due to the evidence of paradoxical herniation. The patient died from sudden death (-Fig. 1).

The present report covers the ethical lineages and the respective authorization from the bioethics committee, as well as the identity protection lineages and data confidentiality.

\section{Discussion}

The decompressive craniectomy was systematically described by Cushing as one of the surgical strategies to diminish the elevated intracranial pressure. Ever since, it has been positioned as an applicable proposition to diverse neurological conditions characterized by mass effect, and the consequent rise in ICP and risk of herniation. ${ }^{3}$
Nowadays the decompressive craniectomy is a strategy applied in an ordinary fashion in many patients that suffer from severe head trauma, acute subdural hematoma, subarachnoid hemorrhage due to a ruptured aneurysm, medial cerebral artery malignant stroke, internal carotid artery stroke, severe cerebral venous thrombosis and dural sinus thrombosis.

Under different strategies, whether it is in form of hemicraniectomy as the most common, or other variants such as the coronal-bifrontal, the objective is to relieve the elevated ICP that has been refractory to the postural, ventilatory, and pharmacological treatments proper of the neurocritique care. ${ }^{7-11}$

In many cases the elevated ICP is controlled, however, the impact in morbi-mortality is still debatable, particularly in the quality of life of the patients submitted to this procedure, mainly referring to the condition of the primary neurological damage, more than the incidents derived from the decompressive craniectomy. The extension of the craniectomy, the topographic variants, the management of the dural opening, the cortical veins management as well as the appropriate time are still debatable, particularly in the long-term results in which survival rates are improved in some cases, but the neurological conditions after survival represent a severe disability. $^{12-14}$

The consequences of removing a part of the skull contribute immediately to the control of the ICP from a biophysical point of view, however, they do not modify per se the primary damage, nor the physiopathological cascade of secondary alterations that have been activated as part of the primary lesion.

The craniectomy by itself may generate associated neurological complications on short, medium, and long terms. All of them have been included in the postcraniectomy syndrome, and clinically are characterized by dizziness, fatigue, headache, psychoemotional, and cognitive conditions, local sensibility or discomfort, pulsating sensation of the brain (specially with postural changes), and the progressive local sinking of the skin flap.
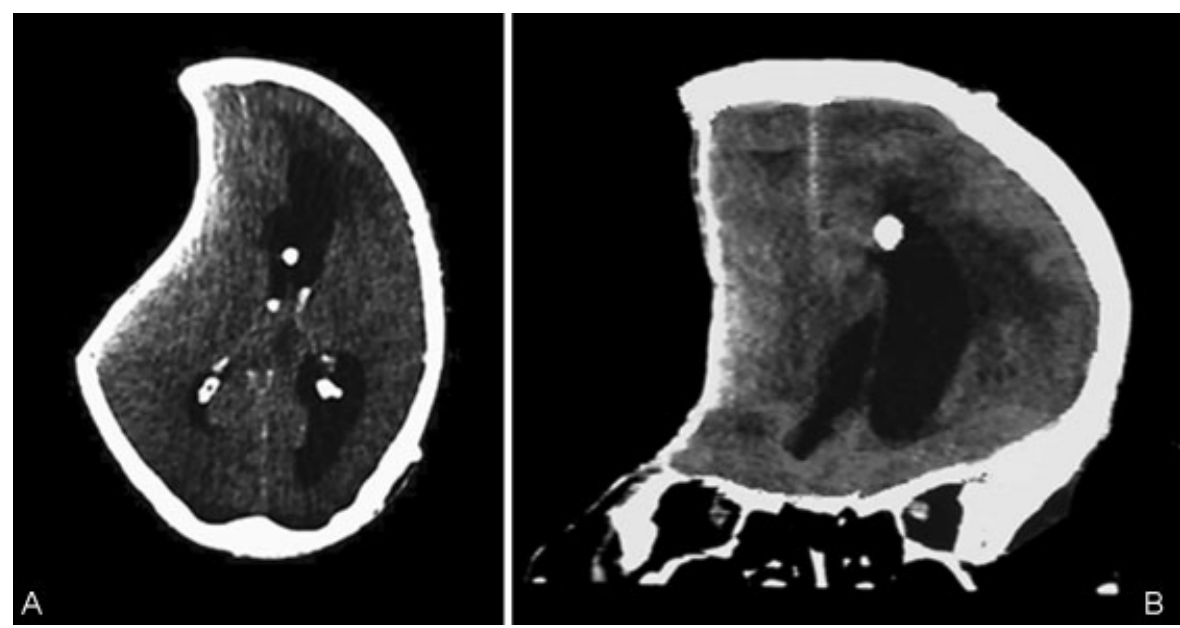

Fig. 1 Simple cranial CT scan (axial and coronal), that shows strong evidence of paradoxical herniation with deviation of the midline, associated findings are the presence of the shunt system and the artifact produced by the aneurysm clip. CT, computed tomographic. 
Some of these complications emerge after a decompressive craniectomy, and are secondary to specific facts such as the presence of ipsilateral hematomas or contralateral to the bone defect, epilepsy, herniation of the cortex along the bone defect in the beginning, leakage of cerebrospinal fluid, infection, subdural spilling, and hydrocephaly. The influence of atmospheric pressure has been recently analyzed in these cases under the description of the syndrome of the trephined, or sinking skin flap syndrome. ${ }^{14-17}$

The absence of a cranium segment alters its equilibrium of the relation content/container and configures changes in the ICP, where the variable of the atmospheric pressure is added as a critical intervening factor. Other studies have demonstrated that in a patient with a major cranial defect, the cerebrospinal fluid pressure in the lumbar region in a vertical position is greater than in a patient with an intact cranial vault. It also showed that after the cranioplasty, the lumbar cerebrospinal fluid pressure returned to its normal levels. The theory suggested to explain these results was that the atmospheric pressure is transmitted to the cranial cavity, inducing the sinking of the skin flap to the interior of the cranial defect. ${ }^{13,14,18,19}$

Other reports talk about a patient subject of multiple lumbar punctures several days after a frontotemporal right craniotomy to relieve her hydrocephaly syndrome, increasing the concavity of the skin flap and as a result the neurological deficit disappeared after the cranioplasty. At the same time, another case was described where a patient developed hydrocephaly after a right frontotemporal craniectomy where a ventriculoperitoneal shunt was placed and this generated an increase of the skin flap concavity of the uncovered area of the skull and cognitive deterioration; both cases improved after cranioplasty. These last two cases show the effects of adding a cerebrospinal fluid leak to patients who previously underwent a craniectomy. ${ }^{14-16}$ This is one of the causes that lead to the sinking of the skin flap, and in the intracranial space results in the paradoxical herniation according to the description of this case.

In all cases, the ideal and timely treatment is the cranioplasty, so it is important to consider that decompressive craniectomy is not harmless, and it presents potential complications secondary to the absence of a portion of the cranial vault and the direct effect of the atmospheric pressure. Such condition generates the alteration in the equilibrium of the content/container intracranial forces and results in the paradoxical herniation. ${ }^{20,21}$

\section{Conclusion}

There is a proactive scenario to increase the number of wide decompressive craniectomies, with no regard for a strict systematization in the inclusion criteria, based on studies with a higher level of evidence. In addition, it is important to consider the full identification of what we now call the postcraniectomy syndrome, also identified in the literature as syndrome of the trephined or sinking skin flap syndrome. This means that the procedure as such is not free from complications and that these complications may present on a short, medium, or long term. The exposed clinical case clearly represents the magnitude of the impact of the atmo- spheric pressure and the effect of the ventriculoperitoneal shunt, as associated factors that intervene in a paradoxical herniation. A timely cranioplasty once the emergency of the mass effect is resolved must be contemplated as mandatory. ${ }^{22}$

\section{Conflict of Interest}

The authors have no conflicts of interests to declare.

\section{Acknowledgment}

None.

\section{References}

1 Hernández I, Matute MC, Abreu F, et al. Decompressive craniectomy in malignant middle cerebral artery infarction. Experience after the implementation of a response protocol [in Spanish]. Rev Neurol 2012;54(10):593-600

2 de Quintana-Schmidt C, Clavel-Laria P, Asencio-Cortes C, VendrellBrucet JM, Molet-Teixido J. Sinking skin flap syndrome [in Spanish].. Rev Neurol 2011;52(11):661-664

3 Waziri A, Fusco D, Mayer SA, McKhann GM II, Connolly ES Jr. Postoperative hydrocephalus in patients undergoing decompressive hemicraniectomy for ischemic or hemorrhagic stroke. Neurosurgery 2007;61(3):489-493, discussion 493-494

4 Uozumi Y, Sakowitz O, Orakcioglu B, et al. Decompressive craniectomy in patients with aneurysmal subarachnoid hemorrhage: a single-center matched-pair analysis. Cerebrovasc Dis 2014;37(2): 109-115

5 Yamaura A, Makino H. Neurological deficits in the presence of the sinking skin flap following decompressive craniectomy. Neurol Med Chir (Tokyo) 1977;17(1 Pt 1):43-53

6 Choi JJ, Cirivello MJ, Neal CJ, Armonda RA. Paradoxical herniation in wartime penetrating brain injury with concomitant skull-base trauma. J Craniofac Surg 2011;22(6):2163-2167

7 Ding J, Guo Y, Tian H. The influence of decompressive craniectomy on the development of hydrocephalus: a review. Arq Neuropsiquiatr 2014;72(9):715-720

8 Fodstad H, Ekstedt J, Fridén H. CSF hydrodynamic studies before and after cranioplasty. Acta Neurochir Suppl (Wien) 1979;28(2):514-518

9 Fodstad H, Love JA, Ekstedt J, Fridén H, Liliequist B. Effect of cranioplasty on cerebrospinal fluid hydrodynamics in patients with the syndrome of the trephined. Acta Neurochir (Wien) 1984; 70(1-2):21-30

10 Gardner WJ. Closure of defects of the skull with tantallum. Surg Gynecol Obstet 1945;80:303-312

11 Grant FC, Norcross NC. Repair of cranial defect by cranioplasty. Ann Surg 1939;110(4):488-512

12 Honeybul S, Ho KM. Incidence and risk factors for post-traumatic hydrocephalus following decompressive craniectomy for intractable intracranial hypertension and evacuation of mass lesions. J Neurotrauma 2012;29(10):1872-1878

13 Jiao QF, Liu Z, Li S, et al. Influencing factors for posttraumatic hydrocephalus in patients suffering from severe traumatic brain injuries. Chin J Traumatol 2007;10(3):159-162

14 Kaen A, Jimenez-Roldan L, Alday R, et al. Interhemispheric hygroma after decompressive craniectomy: does it predict posttraumatic hydrocephalus? J Neurosurg 2010;113(6):1287-1293

15 Langfitt TW. Increased intracranial pressure. Clin Neurosurg 1969; $16: 436-471$

16 Magnaes B. Body position and cerebrospinal fluid pressure. Part 2: clinical studies on orthostatic pressure and the hydrostatic indifferent point. J Neurosurg 1976;44(6):698-705 
17 Romero FR, Zanini MA, Ducati LG, Gabarra RC. Sinking skin flap syndrome with delayed dysautonomic syndromeAn atypical presentation. Int J Surg Case Rep 2013;4(11): 1007-1009

18 Schiffer J, Gur R, Nisim U, Pollak L. Symptomatic patients after craniectomy. Surg Neurol 1997;47(3):231-237

19 Hayward R. Cranioplasty indications, techniques and results. J Neurol Neurosurg Psychiatry 1985;48(5):495
20 Stula D. Intracranial pressure measurement in large skull defects [in German]. Neurochirurgia (Stuttg) 1985;28(4):164-169

21 Winkler PA, Stummer W, Linke R, Krishnan KG, Tatsch K. Influence of cranioplasty on postural blood flow regulation, cerebrovascular reserve capacity, and cerebral glucose metabolism. J Neurosurg 2000;93(1):53-61

22 Kolias AG, Kirkpatrick PJ, Hutchinson PJ. Decompressive craniectomy: past, present and future. Nat Rev Neurol 2013;9(7):405-415 